10-1-1991

\title{
Using Xenon as a Heavy Atom for Determining Phases in Sperm Whale Metmyoglobin
}

Jacqueline Vitali

Cleveland State University, j.vitali@csuohio.edu

Arthur H. Robbins

Miles, Inc.

Steven C. Almo

John Hopkins School of Medicine

Robert F. Tilton

Miles, Inc.

Follow this and additional works at: https://engagedscholarship.csuohio.edu/sciphysics_facpub

Part of the Biophysics Commons

How does access to this work benefit you? Let us know!

Publisher's Statement

(C) 1991 International Union of Crystallography

\section{Original Citation}

Vitali, J., Robbins, A.H., Almo, S.C. and Tilton, R.F. (1991) Using Xenon as a Heavy Atom for Determining Phases in Sperm Whale Metmyoglobin. J. Appl. Cryst. 24, 931-935

\section{Repository Citation}

Vitali, Jacqueline; Robbins, Arthur H.; Almo, Steven C.; and Tilton, Robert F., "Using Xenon as a Heavy Atom for Determining Phases in Sperm Whale Metmyoglobin" (1991). Physics Faculty Publications. 183.

https://engagedscholarship.csuohio.edu/sciphysics_facpub/183

This Article is brought to you for free and open access by the Physics Department at EngagedScholarship@CSU. It has been accepted for inclusion in Physics Faculty Publications by an authorized administrator of

EngagedScholarship@CSU. For more information, please contact library.es@csuohio.edu. 


\title{
Using Xenon as a Heavy Atom for Determining Phases in Sperm Whale Metmyoglobin
}

\author{
By Jacqueline Vitali and Arthur H. RobBins \\ Miles Research Center, Miles Inc., 400 Morgan Lane, West Haven, CT 06516, USA \\ Steven C. Almo \\ Department of Biophysics, Johns Hopkins School of Medicine, 725 North Wolfe Street, Baltimore, \\ MD 21205, USA \\ AND ROBERT F. TILTON \\ Miles Research Center, Miles Inc., 400 Morgan Lane, West Haven, CT 06516, USA
}

(Received 10 August 1990; accepted 14 May 1991)

\begin{abstract}
Xenon gas can be used as a heavy atom for determining phases in a protein. We demonstrate that an interpretable electron density map can be obtained for sperm whale metmyoglobin from a single xenon derivative using iterative single isomorphous replacement with the anomalous scattering method.
\end{abstract}

\section{Introduction}

Xenon is a noble gas with 54 electrons and real and imaginary dispersion corrections for its scattering factor of $f^{\prime}=-0.783, f^{\prime \prime}=7.348 \quad(\mathrm{Cu} \mathrm{K \alpha})$ (International Tables for X-ray Crystallography, 1974). These values suggest that xenon could be useful as a heavy atom for determining phases in proteins.

Several studies suggest that myoglobin has multiple sites for xenon binding. Equilibrium binding studies indicate an equilibrium constant of the order of $200 \mathrm{M}^{-1}$ (Ewing \& Maestas, 1970). NMR studies of ${ }^{129} \mathrm{Xe}$ suggest at least two specific binding sites (Tilton \& Kuntz, 1982). X-ray studies (Schoenborn, Watson \& Kendrew, 1965; Schoenborn, 1968, 1969; Schoenborn \& Nobbs, 1966; Tilton, Kuntz \& Petsko, 1984 ) indicate discrete xenon binding sites with different gas-binding affinities. Further, the gas occupancy of the different sites can be changed by changing the gas pressure. At low pressures [2-3 atm $\left.\left(1 \mathrm{~atm}=1.01 \times 10^{5} \mathrm{~Pa}\right)\right]$ only a single xenon site is observed (Schoenborn, Watson \& Kendrew, 1965), while at higher pressures $(7-10 \mathrm{~atm})$ four sites with fractional occupancies ranging between 1.0 and 0.4 are observed (Tilton, Kuntz \& Petsko, 1984). The xenon binding sites are located within the protein interior and are bordered by the side chains of hydrophobic residues and by the central prosthetic heme group.

0021-8898/91/050931-05\$03.00
In this communication we demonstrate that an interpretable electron density map can be obtained for sperm whale metmyoglobin from a single xenon derivative. This is the first time that xenon has been used as a heavy atom for determining phases in a protein.

\section{Experimental}

Crystals of sperm whale metmyoglobin were grown by the diffusion method, from a solution containing $15 \mathrm{mg} \mathrm{ml}$ ' protein, $66 \%$ saturated ammonium sulfate $\mathrm{pH} 7 \cdot 0$, equilibrated over a reservoir of $77 \%$ saturated ammonium sulfate $\mathrm{pH} 7.0$ at $295 \mathrm{~K}$ (Kendrew, Parrish, Marrack \& Orlans, 1954). The $\mathrm{pH}$ of the ammonium sulfate precipitation agent was adjusted by adding $\mathrm{NaOH}$. A crystal of dimensions $0.6 \times 0.8 \times 1.4 \mathrm{~mm}$ was used for measuring a native data set. A second crystal of dimensions $0.3 \times 0.4 \times$ $0.8 \mathrm{~mm}$ was pressurized with xenon gas (AIRCO, $99.999 \%$ purity) to $\sim 4 \mathrm{~atm}$ and was used for measuring a derivative data set. Both crystals were prismatic in shape.

The procedure used for mounting the crystal that was pressurized with xenon has been described previously (Tilton, Kuntz \& Petsko, 1984) and is only slightly different from standard mounting techniques. Two cotton plugs, moistened with mother liquor, were placed above and below the crystal in the quartz capillary in order to prevent the crystal from moving during the pressurization. The capillary was attached with rubber tubing to a syringe containing xenon gas and flushed. Subsequently, the narrow end was sealed with a microflame torch. The sealed end was then immersed in liquid nitrogen and xenon gas was allowed to distill in. Flame sealing near the top of the capillary and removal from the nitrogen caused pressurization within seconds. Sub-

(c) 1991 International Union of Crystallography 
sequently the flame-sealed ends were coated with ' 5 min' epoxy for extra mechanical stability. Data collection commenced $3 \mathrm{~h}$ after pressurization.

Data were collected at $295 \mathrm{~K}$ on a Rigaku AFC5 diffractometer using monochromatic $\mathrm{Cu} K \alpha$ radiation from a Rigaku RU300 X-ray source operated at $50 \mathrm{kV}$ and $200 \mathrm{~mA}$. The metmyoglobin crystals have space group $P 2$ with cell constants for the native molecule of $a=64.48, b=30.93, c=34.82 \AA, \beta=$ $105.83^{\circ}$ and cell constants for the xenon derivative of $a=64 \cdot 30, b=30 \cdot 72, c=34.71 \AA, \beta=105 \cdot 86^{\circ}$. No additional crystal disorder was caused by the pressurization. Before data collection, reflection shape was examined in both crystals using $\omega$ scans. The reflections were symmetrical with a width at half height of approximately $0 \cdot 25$. Data were collected with the $\omega$-scan technique. The scan speed was $16^{\circ} \mathrm{min}{ }^{\prime}$ for the native and the scan was repeated when $F / \sigma<10$. For the xenon derivative a scan speed of $13^{\circ} \mathrm{min}^{-1}$ was employed with no repeat scans. Friedel pairs were measured every 20 reflections. In each crystal three standard reflections were collected every 400 measurements to monitor the crystal stability and radiation damage. The decay was linear with time and indicated a radiation damage rate in intensity of $0.35 \% \mathrm{~h}{ }^{1}$ for the native over a total of $58 \mathrm{~h}$ and $0.42 \% \mathrm{~h}^{-1}$ for the derivative over a total of $24 \mathrm{~h}$. Lorentz-polarization and decay corrections were applied to the data. Further, the variation of the intensity with $\psi$ for three reflections near $\chi=90^{\circ}$ was used for applying an empirical absorption correction. 17152 independent reflections containing 8153 Friedel pairs to $2.0 \AA$ were measured for the native. 6870 independent reflections containing 2957 Friedel pairs to $2.7 \AA$ were measured for the xenon derivative. Only reflections with $F>2 \sigma$ were used in the subsequent calculations. For data to $2.7 \AA$ Bragg spacing, these constituted $96.9 \%$ of the total for the native and $88.7 \%$ for the xenon derivative. The derivative data were scaled to the native data. Isomorphous and anomalous agreement indices were computed: $R_{\text {iso }}\left(=\sum\left|F_{P H}\right|-\left|F_{P}\right|\left|/ \sum\right| F_{P} \mid\right)=$ $0 \cdot 147, \quad R_{\text {ano,nati }} \quad\left[=\sum|| F_{P}^{+}|-| F_{P}^{-}|| / \sum\left(\left|F_{P}^{+}\right|+\left|F_{P}^{-}\right|\right)\right]$ $=0.017, \quad R_{\text {ano,deri }} \quad\left[=\sum|| F_{P H}^{+}|-| F_{P H}^{-}|| \sum\left(\left|F_{P H}^{+}\right|+\right.\right.$ $\left.\left.\left|F_{P H}^{-}\right|\right)\right]=0.035$, where $\left|F_{P H}\right|$ and $\left|F_{P}\right|$ are the derivative and native structure-factor amplitudes, respectively, and + and - denote the Friedel pairs.

\section{Determination of phases}

The strongest peak in the Harker section $\left(u, \frac{1}{2}, w\right)$ of an isomorphous difference Patterson synthesis computed with data in the resolution range $10 \cdot 0$ to $2 \cdot 7 \AA$ corresponded to the symmetry-related $\mathrm{Xe}-\mathrm{Xe}$ vector and was 4.3 times the height of the next strongest peak in the section (Fig. la). Similarly, the strongest peak in the Harker section of an anomalous difference Patterson synthesis computed with data in the resolution range 10.0 to $3.4 \AA$ corresponded to the symmetry-related $\mathrm{Xe}-\mathrm{Xe}$ interaction and was 1.5 times stronger than the next highest peak in the section (Fig. 1b). At higher resolution, the $\mathrm{Xe}-\mathrm{Xe}$ vector in the Harker section of the anomalous difference Patterson synthesis was at the noise level. The $x$ and $z$ coordinates of xenon were thus determined; $y$ was arbitrarily given the value of 0.865 from the structures of xenon-metmyoglobin at 2.5 atm (Schoenborn, Watson \& Kendrew, 1965) and at 7.0 atm (Tilton, Kuntz \& Petsko, 1984).

The $x$ and $z$ coordinates and the occupancy factor of the xenon site were subsequently refined by the method of origin-removed Patterson correlation (Terwilliger \& Eisenberg, 1983) using data to $3 \cdot 2 \AA$. At the conclusion of the refinement the mean figure of merit was 0.47 . The ratio r.m.s. $\mid F_{H} ; /$ r.m.s. $E$ was 1.17 for centric reflections and 1.35 for acentric

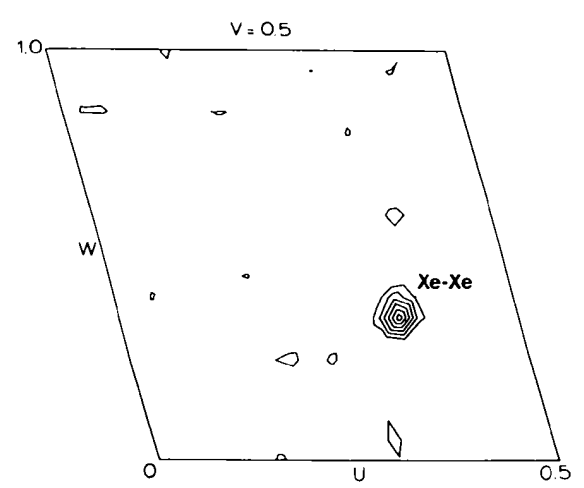

(a)

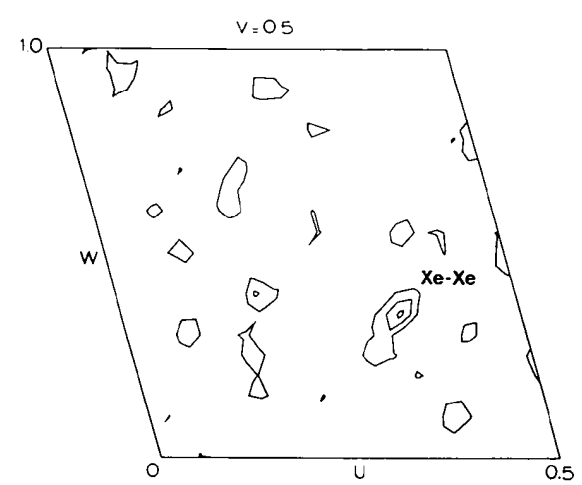

(b)

Fig. 1. (a) Asymmetric unit of the Harker section $\left(u, \frac{1}{2}, w\right)$ of an isomorphous difference Patterson synthesis computed with data in the resolution range $10-2 \cdot 7 \AA$. Contours are in arbitrary units and are drawn at an interval of 15 starting from 15 . Xe-Xe denotes the peak corresponding to the symmetry-related $\mathrm{Xe}-\mathrm{Xe}$ vector. (b) Asymmetric unit of the Harker section $\left(u, \frac{1}{2}, w\right)$ of an anomalous difference Patterson synthesis computed with data in the resolution range $10 \cdot 0-3 \cdot 4 \AA$. Contours are in arbitrary units and are drawn at an interval of 30 starting from 30 . Xe-Xe denotes the peak corresponding to the symmetry-related $\mathrm{Xe}-\mathrm{Xe}$ interaction. 
reflections; $\left|F_{H}\right|$ is the calculated heavy-atom structure factor and $E$ is the lack-of-closure error.

Phases were calculated with the iterative single isomorphous replacement with anomalous scattering method (ISIRAS) (Wang, 1985). SIR and SAS phase probability distributions were calculated using data to 2.7 and $3.4 \AA$, respectively, and subsequently combined with the method of Hendrickson \& Lattman (1970). Two filters were computed with the Wang procedure assuming 30\% solvent content and the phases were refined for five cycles with each. Following phase refinement, the average figure of merit was $0 \cdot 80$. This high value does not necessarily indicate an interpretable electron density map since, in general, the average figure of merit is high after the use of the Wang method. However, as will be seen in the next section, the electron density map was interpretable. It may be noted that the inclusion of the second filter in the phase calculation did not improve the electron density map.

\section{Results and discussion}

A Fourier map was calculated using as coefficients the difference in the structure-factor magnitudes between the xenon-metmyoglobin and metmyoglobin structures and phases determined by the ISIRAS procedure. As expected (Schoenborn, Watson \& Kendrew, 1965), the map was featureless apart from the peak corresponding to the xenon site, indicating that xenon binding did not perturb the molecule and that xenon atoms are not present at subsidiary sites to any appreciable degree at $4 \mathrm{~atm}$ of xenon pressure.

The electron density map was subsequently examined. As seen in Fig. 2, the fit of the known shape of the heme group to this map is reasonable. Further, all helical fragments but helix $F$ were clear and could be fitted using model polyalanine helices. However,
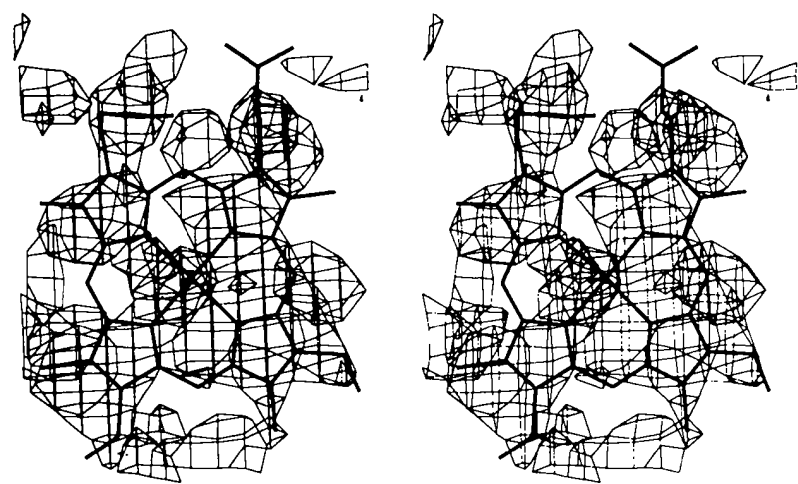

Fig. 2. A stereoview illustrating the fit of the heme group to the electron density. The map was computed using as coefficients the structure-factor magnitudes of the metmyoglobin structure and phases determined by the ISIRAS procedure. Contours are at 0.20 of the maximum density, $1.26 \sigma$. the course of the polypeptide chain was ambiguous at the connections between helices, except between helices $B$ and $C$ and $D$ and $E$. Without prior knowledge of the structure, most of the polypeptide chain would have been correctly interpreted. Fig. 3 shows the $\alpha$-carbon backbone determined from the present ISIRAS calculations superimposed on the known myoglobin structure. The structure of metmyoglobin from sperm whale was first refined by Takano (1977) at $2.0 \AA$ to $R=0.26$ and later by Frauenfelder, Petsko \& Tsernoglou (1979) at $1.5 \AA$ to $R=0.18$. The coordinates from the later refinement, kindly provided to one of us (RFT) by G. A. Petsko, are used for the known metmyoglobin structure in Fig. 3. Fig. 4 shows the fit of a portion of the polyalanine model for helix $E$ to the map.

The idea of using xenon as a heavy atom in protein structure determination was first proposed by Schoenborn \& Featherstone (1967). These authors point out that xenon is a little 'lighter' than desirable

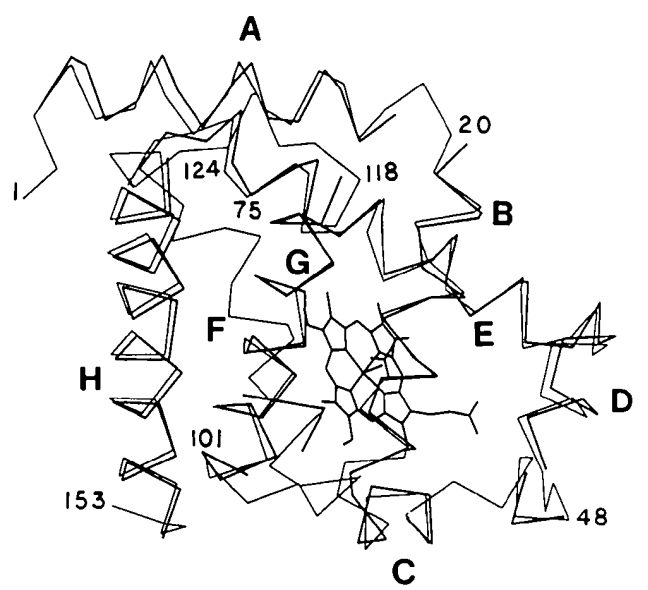

Fig. 3. Heme and the $\alpha$-carbon backbone determined from the ISIRAS calculations (thick lines) superimposed on the known metmyoglobin structure (thin lines). Helices are lettered $A$ through $H$.

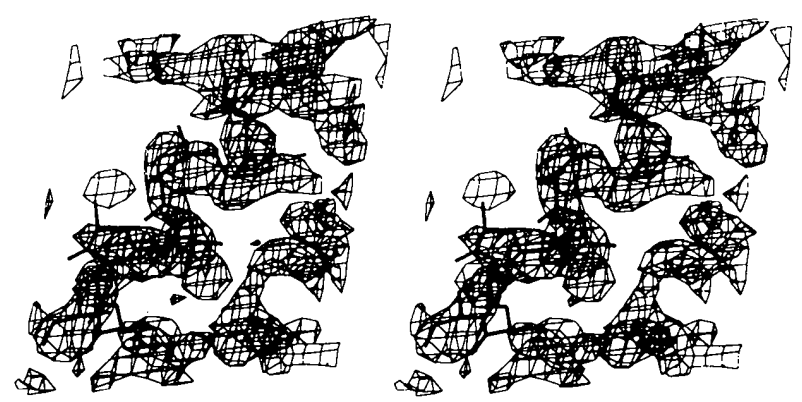

Fig. 4. A stereoview illustrating the fit of a portion of the polyalanine model for helix $E$, residues 65 through 76 , to the electron density map. The map was computed using as coefficients the structure-factor magnitudes of the metmyoglobin structure and phases determined by the ISIRAS procedure. Contours are at 0.20 of the maximum density, $1 \cdot 2 \sigma$. 
for a heavy atom. However, this is counteracted by the fact that xenon-protein complexes show a very high degree of isomorphism with the native crystals. This is often not true with other commonly used heavy atoms which are generally ionic groups capable of inducing some disorder in the native structure. In this communication we demonstrate that the phases determined from a single bound xenon are of sufficient quality to produce an interpretable electron density map for sperm whale metmyoglobin.

The use of xenon in the phase determination of other proteins obviously depends on whether it binds to the protein. In addition to myoglobin, it is known that xenon binds to hemoglobin (Conn, 1961; Tilton \& Kuntz, 1982), each chain having one specific xenon binding site (Schoenborn, 1965), to albumin (Conn, 1961), to rennin (Schoenborn \& Featherstone, 1967) and to the protein subunits of tobacco mosaic virus (Schoenborn, 1968). The studies of xenon binding to myoglobin are extensive and provide some insight into the type of sites to which xenon may bind in other proteins.

Myoglobin has a characteristic arrangement of interior space. As a consequence of its amino acid sequence and tertiary structure, it contains a number of atomic-sized cavities with volumes ranging from 30 to $100 \AA^{3}$ (Tilton, Kuntz \& Petsko, 1984; Tilton, Singh, Weiner, Connolly, Kuntz, Kollman, Max \& Case, 1986). These cavities are distributed throughout the protein interior, but are concentrated where helices pack against other helices or against the planar prosthetic heme group. They are bounded primarily by hydrophobic residues (Leu, Phe, Ile, Val). Xenon is known to bind to certain of these pre-existing cavities with minimal perturbation of the protein structure.

At low pressures (2-3 atm), xenon binds to myoglobin in the proximal cavity opposite the heme group from the $\mathrm{O}_{2}$ binding site. This site was found to be almost fully occupied in metmyoglobin (Schoenborn, Watson \& Kendrew, 1965), deoxymyoglobin (Schoenborn \& Nobbs, 1966) and alkaline myoglobin (Schoenborn, 1969). A second loweroccupancy xenon site was also observed in alkaline myoglobin in the $A-B, G-H$ corner region. At higher pressures $(7-10 \mathrm{~atm})$, four xenon sites are observed (Tilton, Kuntz \& Petsko, 1984). One xenon is bound in the proximal pocket, as at low pressures, and has essentially full occupancy. The other three xenon sites have lower occupancies. They are located in a relatively small cavity near the bottom of the heme group directly below the proximal cavity, a cavity formed by the $E-F$ helix corner and the $H$ helix near the surface of the protein and in the distal cavity directly below the oxygen binding site.

Schoenborn, Watson \& Kendrew (1965) suggested that the binding of xenon to the proximal pocket is stabilized by dispersion interactions, charge-induced dipole moments and dipole-induced dipole moments, since the xenon atom in this cavity lies between a nonpolar area and an area which is partially polar. It is in contact with the heme-linked histidine, a pyrrole ring of the heme group and residues Leu 89, Ala 90, Leu 104, Phe 138 and Ile 142. The dispersion interaction was estimated to be $42 \mathrm{~kJ} \mathrm{~mol}^{-1}$. More recent empirical energy calculations (Tilton, Singh, Weiner, Connolly, Kuntz, Kollman, Max \& Case, 1986) indicated a favorable enthalpic contribution of 2.5 to $17.5 \mathrm{~kJ} \mathrm{~mol}^{-1}$ to xenon binding for the four experimentally determined xenon sites at higher pressures (Tilton, Kuntz \& Petsko, 1984). These results are in agreement with the different xenon occupancies observed experimentally. The most attractive enthalpic terms were found to be dispersion interactions, while polarization terms were relatively minor.

The foregoing discussion suggests that xenon could potentially bind to other proteins containing hydrophobic cavities. These hydrophobic pockets may be naturally present in the protein as a consequence of the protein fold and amino acid sequence, as is the case in myoglobin. Also, they may be transient, created by factors such as crystallization conditions or application of xenon pressure. For instance, as discussed earlier in this section, at 2-3 atm of xenon pressure alkaline myoglobin binds a second xenon atom with lower occupancy in the $A-B, G-H$ corner region (Schoenborn, 1969). Tilton, Kuntz \& Petsko (1984) discuss that alkaline conditions deprotonate His 119 in myoglobin leading to protein structural changes in the $A-B$ and $G-H$ corners as well as in the $E$ helix and the creation of a new cavity capable of binding an $\mathrm{Xe}$ atom. At $35 \mathrm{~atm}$ of xenon gas, a buried Phe 138 of metmyoglobin moves to a new conformation (Tilton, unpublished results). This conformational rearrangement eliminates one binding site observed in the native structure and creates a new binding site. An interesting point to consider for proteins that do not contain hydrophobic pockets is site-specific mutagenesis. We expect that changes in the atomic distribution near a cavity that create a nonpolar environment can change the interaction with xenon toward an energetically favorable state. Further, sitespecific mutagenesis may provide a means for repacking the protein interior to create new cavities.

This work was supported by NIH grant GM38758-03. JV thanks Dr M. J. Colaneri for discussions on cryogenic techniques.

\section{References}

Conn, H. L. (1961). J. Appl. Physiol. 16, 1065-1070.

Ewing, G. J. \& Maestas, S. (1970). J. Phys. Chem. 74, 2341-2344. 
Frauenfelder, H., Petsko, G. A. \& Tsernoglou, D. Schoenborn, B. P. \& Nobbs, C. L. (1966). Mol. Pharma(1979). Nature (London), 280, 558-563. col. 2, 491-498.

Hendrickson, W. H. \& Lattman, E. E. (1970). Acta Cryst. B26, 136-143.

International Tables for X-ray Crystallography (1974). Vol. IV, p. 150. Birmingham: Kynoch Press. (Present distributor Kluwer Academic Publishers, Dordrecht.)

Kendrew, J. C., Parrish, R. G., Marrack, J. R. \& Orlans, E. S. (1954). Nature (London), 174, 946-949.

SCHOENBORN, B. P. (1965). Nature (London), 208, 760-762.

Schoendorn, B. P. (1968). Fed. Proc. Fed. Am. Soc. Exp. Biol. 27, 888-894.

SChOENBORN, B. P. (1969). J. Mol. Biol. 45, 297-303.

Schoenborn, B. P. \& Featherstone, R. M. (1967). $A d v$. Pharmacol. 5, 1-17.

Schoenborn, B. P., Watson, H. C. \& Kendrew, J. C. (1965). Nature (London), 207, 28-30.

Takano, T. (1977). J. Mol. Biol. 110, 537-568.

Terwilliger, T. C. \& EisenberG, D. (1983). Acta Cryst. A39, 813-817.

Tilton, R. F. \& Kuntz, I. D. (1982). Biochem. 21, 6850-6857.

Tilton, R. F., Kuntz, I. D. \& Petsko, G. A. (1984). Biochemistry, 23, 2849-2857.

Tilton, R. F., Singh, U. C., Weiner, S. J., Connolly, M. L., Kuntz, I. D., Kollman, P. A., MaX, N. \& Case, D. A. (1986). J. Mol. Biol. 192, 443-456.

WANG, B.-C. (1985). Methods Enzymol. 115, 90-112. 